\title{
Perspectivas de las tecnologias de Chatbot y su aplicación a las entrevistas de evaluación del lenguaje
}

\section{Mario Crespo Miguel}

Área de Lingüística General

Laboratorio de Lingüística Computacional y

digital

Instituto de Investigación en Lingüística

Aplicada

Universidad de Cádiz

Avenida Duque de Nájera, 16, Edificio Simón Bolivar, $2^{a}$ planta

11002 Cádiz

E-mail: mario.crespo@uca.es

\author{
Berenice domínguez Cabrera \\ Gabinete de Lingüística Clínica \\ Instituto de Investigación en Lingüística \\ Aplicada \\ Universidad de Cádiz \\ Avenida Duque de Nájera, 16, \\ Edificio Simón Bolívar, $2^{\mathrm{a}}$ planta \\ 11002 Cádiz \\ E-mail: berenice.dominguez@uca.es
}

PERSPECTIVAS DE LAS TECNOLO GÍAS DE CHATBOT Y SU APLICACIÓN A LAS ENTREVISTAS DE EVALUACIÓN DEL LENGUAJE

RESUMEN: Un chatbot puede considerarse como un sistema pregunta-respuesta basado en el conocimiento que una serie de expertos le han proporcionado de antemano. Los chatbots son agentes artificiales diseñados para implicarse en conversaciones de texto (tipo chat) con interlocutores humanos. Son programas que intentan emular la conversación humana usando Inteligencia Artificial. La literatura científica sobre el tema refleja un creciente interés actualmente en ámbitos como la medicina, la enseñanza y los negocios. Este artículo explora los usos médicos de estas nuevas tecnologías, su composición, para posteriormente analizar su aplicabilidad a la mejora del proceso de entrevistas enfocadas en la evaluación del lenguaje. Este tipo de tecnologias podría establecer una comunicación constante con el paciente y sus familiares adaptándose a sus características sociolingüísticas y culturales, favoreciendo su participación y su colaboración en el mismo. Las perspectivas de aplicabilidad son prometedoras.
PERSPECTIVES OF CHATBOT TECHNOLOGIES AND THEIR APPLICATION TO LANGUAGE ASSESSMENT INTERVIEWS

ABSTRACT: A chatbot can be considered as a question-answer system based on the knowledge that a series of experts have provided previously. Chatbots are artificial agents designed to engage in text conversations (chat-like) with human interlocutors. They are programs that attempt to emulate human conversation using Artificial Intelligence. The scientific literature on the subject reflects a growing interest today in fields such as medicine, teaching and business. This article explores the medical uses of these new technologies, their composition, and then analyses their applicability to the improvement of the process of interviews on language evaluation. This type of technology could establish a constant communication with the patient and his relatives, adapting itself to their sociolinguistic and cultural characteristics, fostering their participation and collaboration. The perspectives of applicability are promising.
PALABRAS ClAVES: Chatbots; Agentes conversacionales; Logopedia; Evaluación del Lenguaje; Telemedicina.

SUMARIO: 1. Introducción. 2. Chat bots, Chatterbots o agentes conversacionales. 3. Tecnologia del chatbot: el formato AIML en breve. 4. Aplicabilidad del Chatbot a la entrevista clínica par la evaluación del lenguaje. 5. Conclusiones.
KEY WORDS: Chatbots; Conversational Agents; Speech Therapy; Language Evaluation; Telemedicine.

SUMMARY: 1 . Introduction. 2. Chatbots, Chatterbots or conversational agents. 3. Chatbot technology: the AIML format in brief. 4. Applicability of Chatbot to clinical interview for language assessment. 5. Conclusions.

\section{PERSPECTIVES DES TECHNOLOGIES CHATBOT ET LEUR APPLICATION AUX ENTRETIENS D'EVALUATION LINGUISTIQUE}

RÉSUMÉ: Un chatbot peut être considéré comme un système de questions-réponses basé sur les connaissances qu'une série d'experts ont fourni au préalable. Les chatbots sont des agents artificiels conçus pour engager des conversations textuelles (de type chat) avec des interlocuteurs humains. Ce sont des programmes qui tentent d'imiter la conversation humaine en utilisant l'intelligence artificielle. La littérature scientifique sur le sujet reflète un intérêt croissant aujourd'hui dans des domaines tels que la médecine, l'enseignement et les affaires. Cet article explore les utilisations médicales de ces nouvelles technologies, leur composition, pour ensuite analyser leur applicabilité à l'amélioration $\mathrm{du}$ processus des entretiens destinés à l'évaluation linguistique. Ce type de technologie pourrait établir une communication constante avec le patient et sa famille, en s'adaptant à leurs caractéristiques sociolinguistiques et culturelles, en favorisant leur participation et leur collaboration dans la même. Les perspectives d'applicabilité sont prometteuses.

MOTS CLÉS: Chatbots; Agents conversationnels; Orthophonie; Évaluation du langage; Télémédecine

SOMMAIRE: 1 . Introduction. 2 Chatbots, Chatterbots ou agents conversationnels. 3 . Technologie Chatbot: le format AIML en bref. 4 Applicabilité de Chatbot à l'entrevue clinique pour l'évaluation linguistique. 5. Conclusions 


\title{
Perspectivas de las tecnologias de Chatbot y su aplicación a las entrevistas de evaluación del lenguaje
}

\author{
Mario CRespo Miguel \& Berenice Domínguez CABrera
}

\section{INTRODUCCIÓN}

La importancia de las nuevas tecnologías de la comunicación e información es evidente en la sociedad actual. Cada vez estamos más acostumbrados a la búsqueda de contenidos y de servicios a través de la web, así como a la inmediatez de la información y a la facilidad de uso. En este contexto, organismos sociales y culturales buscan nuevas formas de comunicarse y de poner en valor sus bienes y servicios. De esta manera, hoy en dia es bastante común que tanto empresas como grupos políticos y socioeconómicos muestren sus productos a través de la web, así como que dispongan de perfiles en redes sociales con vistas a su difusión, la ampliación de su mercado y no quedarse al margen del intercambio de información y del contacto permanente independientemente de la ubicación geográfica (Boshmaf et al., 2011; Pollak y Anderst-Kotsis, 2017).

La Lingüística computacional es una rama interdisciplinar de la Lingüística implicada en el análisis y generación automática del lenguaje humano. Algunos lingüistas consideran que la Lingüistica computacional es un campo del saber que relaciona lenguas naturales y ordenadores (Sandoval, 1998: 13). El objetivo de la Lingüística computacional es desarrollar teorías lingüísticas computables, es decir, que puedan ser aplicadas sutilmente a los ordenadores (León Pérez et al., 2017). Puede ser entendida desde dos puntos de vista; uno, relacionado con la informática, donde suele ser considerada como una subdisciplina de la Inteligencia Artificial; el otro relaciona la Lingüística computacional con la Lingüística, es decir, la considera una subdisciplina de la Lingüística teórica (Guinovart, 1998: 135). Dentro de la Lingüistica computacional, un chatbot es un sistema informático que puede conversar con un ser humano usando su propia lengua (AbuShawar y Atwell, 2015). En su forma usual, el chatbot es una aplicación de software que permite crear un hilo conversacional con el usuario (Guerrero et al., 2017). Aunque, en sus inicios, estaban limitados a la experimentación, con el uso masivo de internet su empleo se está extendiendo a los negocios (Plaza Navas, 2017; Thomas, 2016), la sanidad (Abashev et al., 2017; Mishra et al., 2018) o la educación (Bii, 2013; Harvey et al., 2016; Delgado Guerrero et al., 2017).

El desarrollo de estas aplicaciones está siendo vertiginoso. Entre 2007 y 2015 , los chatbots participaron entre un tercio y la mitad de las interacciones que se produjeron online y esta tasa se está incrementando desde entonces (Radziwill y Benton, 2017). Actualmente su gran eclosión se debe a 
su unión a las redes sociales (Pollak y Anderst-Kotsis, 2017). De esta manera, para el gigante de la informática Oracle (2017), ya existen actualmente unos 30.000 bots de negocios entre Facebook y Kik con el objetivo de acelerar y acortar trámites, aportar, recopilar y ayudar en la gestión de los usuarios y clientes las veinticuatro horas del dia con total disponibilidad.

Este artículo explora los posibles usos médicos actuales de estas nuevas tecnologias, su composición, para posteriormente analizar su aplicabilidad en la evaluación del lenguaje. En el siguiente apartado, exploraremos la composición de este tipo de tecnologias.

\section{Chatbots, Chatterbots o Agentes Conversacionales}

Un chatbot puede considerarse como un sistema pregunta-respuesta basado en el conocimiento que una serie de expertos le han proporcionado de antemano (Deshpande et al., 2017). Para Thorne (2017), los chatbots son agentes artificiales diseñados para implicarse en conversaciones de texto (tipo chat) con interlocutores humanos. Son programas que intentan emular la conversación humana usando Inteligencia Artificial (Ranoliya et al., 2017). Los nuevos chatbots o también socialbots son autómatas que controlan la cuenta de una determinada red social y pueden realizar actividades básicas como mandar y responder a mensajes. La característica determinante de estos chatbots es que son capaces de hacerse pasar por seres humanos (Boshmaf et al., 2011).

De entre todos los agentes de conversación, debemos destacar a ALICE ${ }^{1}$ (Artificial Linguistic Internet Computer Entity), creada por Richard Wallace en 1995 y que, tras varios años de perfeccionamiento, ganó el premio Loebner $^{2}$ en los años 2000, 2001 y 2004. Tras varias versiones previas, en noviembre de 2000 se crea el conocido como "program D", que se ha convertido en el estándar de referencia de ALICE con código abierto, y es usado por la comunidad científica para el desarrollo de aplicaciones. Actualmente existen diferentes versiones dependiendo del lenguaje de programación ${ }^{3}$. ALICE también añade un nuevo rol de botmaster (administrador de la base del conocimiento) que es imprescindible. Este analiza los logs de los diálogos, identifica las mejorias necesarias y crea nuevos contenidos o conocimientos en forma de archivos AIML para que las siguientes respuestas sean más apropiadas.

\footnotetext{
1 https://www.pandorabots.com/pandora/talk?botid=a847934aae3456cb. En este enlace se puede encontrar una versión de Alice adaptada a español a través de la plataforma pandorabots.

2 El Premio Loebner es una competición de carácter anual que concede premios a un programa de ordenador considerado como el más inteligente de los que se han presentado. El formato de la competición sigue el estándar establecido en el test de Turing. http://www.aisb.org.uk/events/loebner-prize.

${ }^{3}$ Este proyecto usa, por ejemplo, el Program O https:/program-o.com, una derivación de ese program $D$ pero para el lenguaje PHP más compatible con el desarrollo de aplicaciones web.
} 
La literatura científica sobre el tema de estos chatbots refleja una gran efervescencia actualmente ya que la mayoria de los artículos se concentran en los últimos 3 o 4 últimos años. Como ya comentábamos en la introducción, la implementación de estos agentes conversacionales se concentra en la enseñanza, los negocios y la medicina. Respecto a su aplicación en educación, se caracterizan por su capacidad para dar información. Entre ellos podemos destacar los trabajos de Delgado Guerrero et al. (2017) que presentan el diseño y desarrollo de un chatbot para ser usado por los estudiantes de la carrera de Ingeniería en Sistemas Computacionales de la Facultad de Ciencias Matemáticas y Físicas de la Universidad de Guayaquil, Ecuador; el trabajo de Ranoliya et al. (2017), que presentan un sistema que está siendo desarrollado en el ámbito de la educación universitaria y al que se le puede pedir información sobre la admisión, la vida de la universidad, aspectos académicos, servicios, etc. y, en el ámbito de la medicina educativa, queremos resaltar el chatbot desarrollado por Kazi et al. (2012) para estudiantes de Medicina. Este chatbot se incorpora a un sistema de tutoría e integra contenidos de $\mathrm{UMLS}^{4}$, un recurso médico que integra el vocabulario de las ciencias biomédicas con más de dos millones de entradas.

Los chatbots dedicados a los negocios no se encuentran muy reflejados en la literatura científica, ya que muchos de ellos se están desarrollando de manera privada. No obstante, encontramos algunos ejemplos de su difusión científica. En el ámbito de los negocios podemos destacar el banking bot project (Dole et al., 2015), dedicado a entender los mensajes y preguntas de los usuarios sobre un banco tales como tipos de préstamos, estado de la cuenta, política de la empresa, etc. Además, el chatbot es capaz de colaborar con el cliente hasta llegar a la información que necesita, dar información adicional sobre productos e intentar satisfacer las necesidades del cliente. Thomas (2016) desarrolla un sistema enfocado al e-business que intenta responder a las preguntas más frecuentes realizadas por los clientes. Finalmente, Plaza Navas (2017) plantea la creación de un chatbot para la gestión interna de la empresa y no necesariamente a clientes, orientándose a la gestión de incidentes informáticos.

Ya en el ámbito médico encontramos a Pharmabot (Comendador et al., 2015), que desarrolla un agente conversacional que actúa como un supuesto "farmacéutico" al que se le puede preguntar sobre medicamentos genéricos y su uso. Por su parte, Kumar et al. (2016) crean un sistema para responder a las preguntas mandadas por sus pacientes sobre salud. Abashev et al. (2017) reflexionan sobre las condiciones que deben cumplir los chatbots en contextos médicos, mientras que Madhu et al. (2017) proponen un sistema de diálogo que pueda, por un lado, ayudar a predecir enfermedades a partir de una lista de síntomas, y por otro, ofrecer una lista de tratamientos disponibles, la composición de las medicinas y sus usos prescritos. De estos

\footnotetext{
${ }^{4}$ https://www.nlm.nih.gov/research/umls/. El UMLS integra y distribuye terminología clave, normas de clasificación y codificación, asi como recursos asociados para promover la creación de sistemas y servicios de información biomédica más eficaces e interoperables.
} 
trabajos médicos, por temática, destaca el de Cameron et al. (2017), cuyo objetivo es el de crear un chatbot para ayudar en terapias de salud mental. Ya en 2018, Mishra et al. se plantean la creación de un chatbot que actuará como doctor virtual para que los pacientes pueden interactuar con él y Divya et al. (2018) proponen un sistema que incluso sea capaz de proveer detalles sobre la enfermedad al paciente antes de la consulta con el doctor real.

En el siguiente apartado veremos el funcionamiento interno de este tipo de tecnología. La implementación técnica del bot no posee gran complejidad técnica, siendo la formalización y análisis lingüístico los aspectos en donde realmente radica su complejidad (Augello et al., 2017).

\section{TECNOLOGÍA DEL CHATBOT: EL FORMATO AIML EN BREVE}

Como ya explicamos anteriormente, ALICE almacena conocimiento sobre patrones de conversación AIML, un derivado de XML desarrollado por la comunidad de software libre de Alicebot durante 1995-2000. AIML consiste en objetos de datos formados por unidades llamadas categorías. Estas son las unidades básicas de conocimiento de AIML (AbuShawar y Atwell, 2015; Satu y Parvez, 2015; Wallace, 2009). Cada categoría es una regla para emparejar una entrada y convertirla en una salida y consiste en un patrón o input de entrada (pattern), que representa la entrada del usuario, y una plantilla u output (template), que implica la respuesta del robot. En la figura 1 se puede observar un ejemplo de archivo AIML que prevé, si el usuario escribe "hola" al bot, la respuesta "Hola, ¿qué tal?".

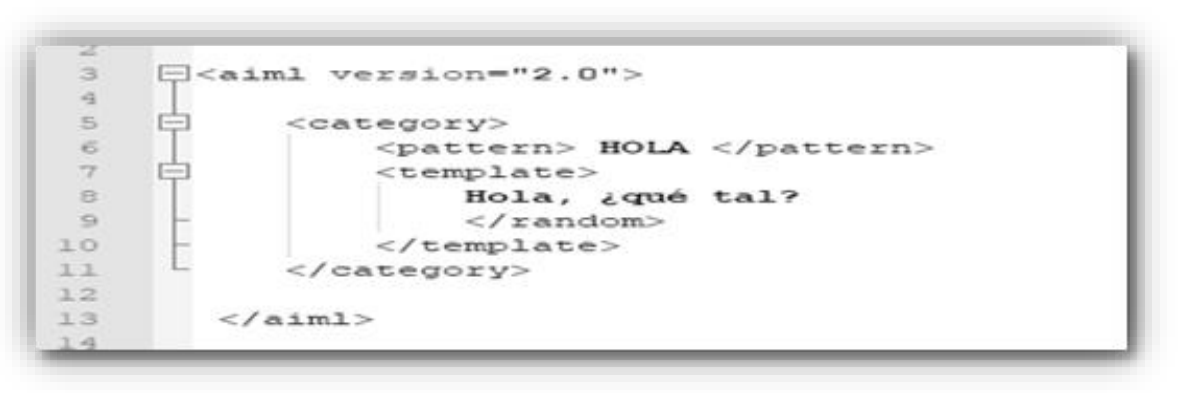

Figura 1: Representación básica de AIML

Como se puede observar, el formato patrón de AIML es extremadamente simple. Las etiquetas básicas de AIML son las siguientes:

\begin{tabular}{|c|c|}
\hline ETIQUETAS & DEFINICIÓN \\
\hline$<$ AIML $>$ & $\begin{array}{l}\text { Etiqueta de apertura y cierre de AIML que engloba el resto } \\
\text { del código }\end{array}$ \\
\hline$<$ CATEGORY $>$ & $\begin{array}{l}\text { Unidad de conocimiento: contiene una pregunta y una res- } \\
\text { puesta }\end{array}$ \\
\hline <PATTERN $>$ & Define un patrón que dé coincidencia \\
\hline
\end{tabular}


Figura 2: Principales etiquetas en la codificación de conversaciones

El funcionamiento del sistema con los archivos AIML es bastante sencillo y queda reflejado en el siguiente cuadro:

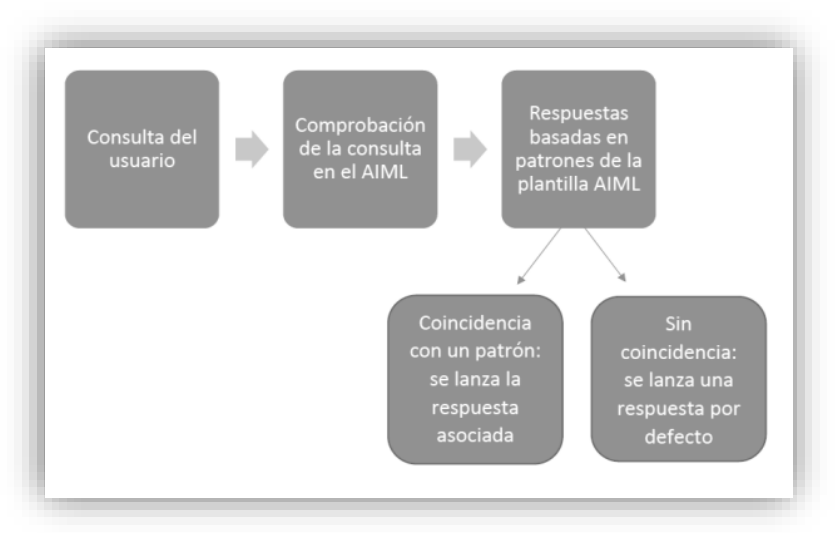

Figura 3: Funcionamiento de un sistema con AILM

La figura anterior muestra el funcionamiento de este tipo de plataformas. Una vez que se produce la consulta del usuario, el sistema comprueba si este patrón de entrada está recogido en su base de conocimiento. El intérprete de AIML intenta emparejar palabra por palabra para conseguir la coincidencia de patrón más larga. La idea de la técnica de emparejamiento de patrones se basa en encontrar la coincidencia de patrón más largo y mejor. Si encuentra una coincidencia, lanza la respuesta asociada, en caso contrario muestra una respuesta por defecto. El sistema procede en una secuencia de búsqueda de "primer nivel". Si no encuentra una coincidencia, el algoritmo de búsqueda retrocede al último nodo con ramas inexploradas y busca esas (Wei et al., 2016).

La estructura básica de AIML contempla el uso de otros elementos que mejoran las capacidades de comprensión y respuesta del bot. En primer lugar, el input de entrada se caracteriza por contener palabras (letras y números, pero no otro tipo de caracteres), sin embargo, este se puede enriquecer con el uso de comodines: guion bajo '_, , circunflejo '^' o estrellas '*'.

\begin{tabular}{|c|c|}
\hline ETIQUETAS & DESCRIPCIÓN \\
\hline$*$ & Empareja una o más palabras \\
\hline- & Empareja una o más palabras, tiene prioridad sobre otros comodines \\
\hline$\wedge$ & Empareja ninguna o más palabras \\
\hline
\end{tabular}

Figura 4: Comodines lingüísticos en la codificación de conversaciones 
Junto con las etiquetas anteriores, existen elementos opcionales. Entre ellos queremos resaltar por su funcionalidad "that", "srai" y "li". "That" almacena la última expresión del chatbot, "srai" permite repetir la misma respuesta a diferentes inputs y "li" permite la selección aleatoria de una respuesta entre un conjunto de ellas almacenadas. De acuerdo con Satu y Parvez (2015), las categorias se AIML se clasificarian básicamente en tres tipos:

1. Categorias atómicas: estas categorias son patrones que no tienen comodines.

2. Categorias predeterminadas: estas categorias son aquellas que contienen comodines (“' y ' ${ }^{\text {(*) }}$ ) que se pueden emparejar con cualquier cadena.

3. Categorias recursivas: estas categorias son aquellas que tienen etiquetas <srai > de recuperación de información.

Existen muchas otras etiquetas con funciones menores que le otorgan mayor realismo y expresividad al lenguaje AIML. Se trata de un sistema simple, aunque muy efectivo (Thorne, 2017), que, como dijimos, le ha valido el Premio Loebner en tres ocasiones. Entre las ventajas de AIML, se encuentra el hecho de que muchos archivos AIML con diferentes áreas de conocimiento y de conversación se pueden combinar en un corpus para mejorar la escalabilidad y compatibilidad del sistema. Entre sus desventajas está el hecho de que el desarrollador tiene que escribir los patrones para todos los inputs que el sistema puede recibir (Thomas, 2016), lo que puede ser una tarea complicada.

La competencia semántica del lenguaje AIML es bastante débil. Si una pregunta o petición se puede formular de muchas maneras, deberán estar recogidas todas, de ahí su complejidad lingüística. Por lo tanto, es muy importante el proceso de enriquecimiento lingüístico del corpus AIML. Las consultas de los usuarios pueden considerarse en última instancia como objetos con una estructura léxico-semántica particular (AbuShawar y Atwell, 2015). Estos objetos deben recuperarse de un conjunto de registros posibles almacenados en una base de datos (DB). González Moreno et al. (2010) explican que ALICE implementa el modelo de aprendizaje supervisado, en el cual el papel del botmaster (administrador de la base de conocimiento) es fundamental, pues analiza los logs de los diálogos, identifica las mejorias necesarias y crea nuevos contenidos o conocimientos en forma de archivos AIML para que las siguientes respuestas sean más apropiadas. Este tipo de desarrollos y análisis lingüístico-comunicativos pueden ser complicados para un desarrollador informático, pero no para un lingüista. 


\section{Aplicabilidad del Chatbot a la entrevista Clínica para la eVAluación DEL LENGUAJE}

Como se puede apreciar, los bots conversacionales sociales pueden usarse para beneficiar a instituciones, reducir el tiempo de respuesta, proporcionar un mejor servicio a clientes, estudiantes y pacientes, aumentar la satisfacción y aumentar la participación (Radziwill y Benton, 2017). En su forma más simple, un chatbot podría guiar al usuario, transformar la búsqueda web a una cuasi-conversación como forma más propicia para obtener información de los usuarios con respecto a sus deseos y necesidades (D'Alfonso et al., 2017). Este tipo de aplicaciones son adecuadas para aquellas situaciones donde la conversación cobra una especial importancia.

En el ámbito de la logopedia, antes de realizar cualquier tipo de intervención, asesoramiento o prevención del lenguaje es necesario realizar una evaluación. De ahí su importancia, puesto que nos va a determinar y condicionar las actuaciones futuras. El primer paso que realiza el terapeuta es preguntarse qué evaluar y cómo evaluar. Para responderse a estas preguntas el logopeda considera la entrevista inicial como un instrumento básico de trabajo y como una toma de contacto inicial (Rodríguez Boggia, 2006). La entrevista clínica es una conversación organizada que estará diseñada para recopilar cualquier tipo de detalle sobre el paciente y definir al máximo todos los datos pertinentes. Todo lo que sucede durante la anamnesis tendrá su repercusión en el resto del proceso evaluador y, posteriormente si es el caso, en la rehabilitación (Pérez Montero, 1995; Peña-Casanova, 1996; Rodríguez Morejón, 1997).

Asimismo, el uso de estas tecnologías de la conversación nos permite aplicarlos en diferentes ámbitos de la evaluación logopédica:

1. Dar información general sobre alguna patología. Se trata este del uso normal de los chatbots en la literatura científica sobre el tema (Comendador et al., 2016; Kumar et al., 2016; Abashev et al., 2017; Cameron et al., 2017; Divya et al., 2018). De este modo, el bot podría ejercer las funciones de un glosario logopédico ofreciendo definiciones sobre patologías, información sobre algún término de este ámbito, etc. Así como ofrecer consejos, asesoramiento y recomendaciones: higiene vocal, estimulación del lenguaje, pautas para el entorno familiar... Como se aprecia en la Figura 5, se puede implementar muy fácilmente un glosario médico en el que el paciente puede preguntar por determinadas enfermedades. 


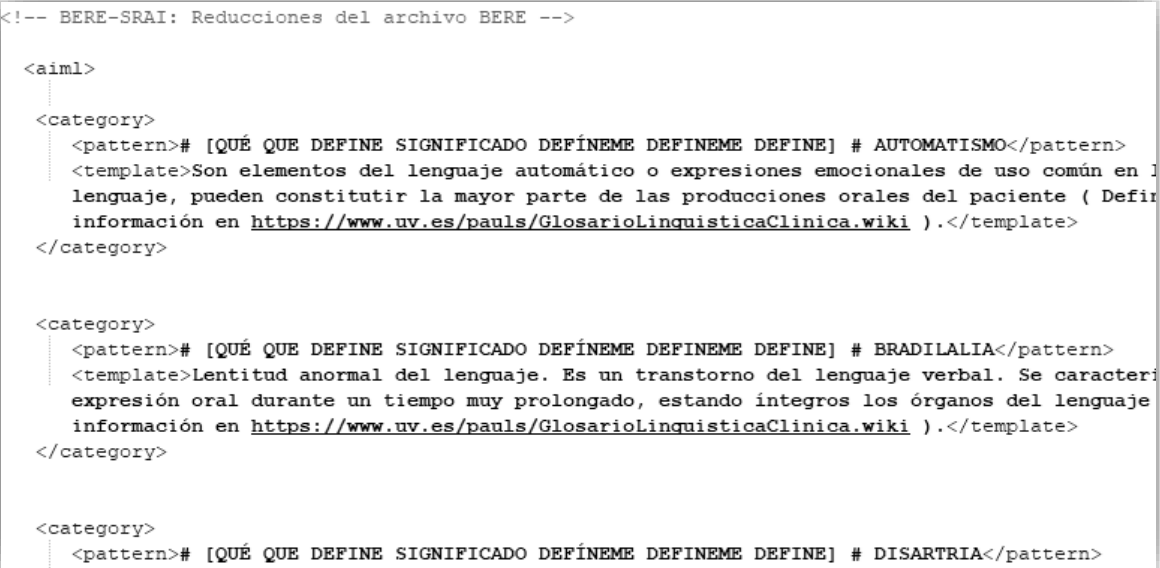

Figura 5: Implementación de conocimiento médico en AIML

2. Ser un primer contacto con pacientes o familiares como complemento a la evaluación. La interacción con el chatbot podría ser una primera toma de contacto para la obtención de información del paciente y, sobre todo, de cómo ha sido su desarrollo en diversas áreas: motora, lenguaje, cognición, sociabilización, escolari-

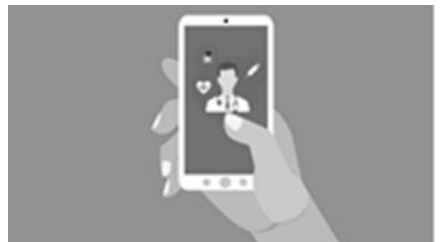

Figura 6: Interacción del paciente remotamente zación, etc. Permite un acercamiento cómodo para aquellos pacientes que sean reticentes a acudir a un logopeda directamente y puede aportar un gran ahorro de tiempo. En la Figura 6 podemos observar la interacción del usuario con la aplicación desde el lugar y el momento que le resulte más cómodo.

3. Proporcionar o ser fuente parcial de evaluación de manera remota o telemédica. El bot nos aporta una gran cantidad de datos para que el evaluador estructure la evaluación, como hemos mencionado con anterioridad, y nos permiten implementar en este tipo de agentes conversacionales cuestionarios evaluadores enfocados a una patología concreta entre los que encontramos Rosseti

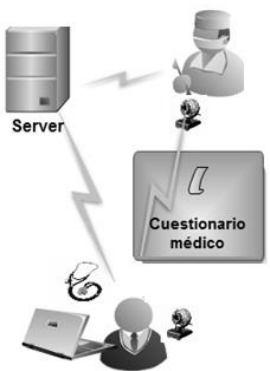

Figura 7: Esquema básico telemédico 
(2006), Wetherby y Barry (2002), Dewart y Summers (1988), Robins (2009), Ehlers et al. (1999), Riviere (2004), Fernández-Zuñiga (2005), Borragán et al. (1999). En la Figura 7 se muestra el esquema básico del funcionamiento telemédico donde los datos del paciente se guardan en un servidor que es accesible por el médico para seguir la evolución del paciente.

De este último punto se derivan las siguientes aplicaciones:

4. Rastreos masivos. Tanto en el ámbito escolar, los equipos de orientación educativa pueden detectar posibles alteraciones o retrasos mediante la interacción con el bot de los alumnos, padres y tutores. Como en el sector sanitario donde los usuarios con patología vocal pueden usar esta tecnología en la consulta del otorrinolaringólogo para ejecutar las posibles derivaciones al técnico especialista en logofoniatría.

5. Accesibilidad. Este tipo de plataformas pueden ayudar a personas con limitaciones sensoriales, culturales o generacionales a acceder a aplicaciones tecnológicas, lo que permite una mayor inclusión social de sectores vulnerables de la sociedad.

\section{Discusión Y CONCLUSIONES}

Es evidente que los chatbots están despertando un gran interés en contextos que necesitan proporcionar información a los usuarios, los clientes, los estudiantes o los pacientes. La aplicación de los bot en el ámbito logopédico puede ser una actualización y un complemento a los métodos tradicionales de evaluación del lenguaje, concretamente, a la entrevista inicial y a la anamnesis, pudiendo permitir al logopeda un gran ahorro de tiempo para que organice y planifique la evaluación en sí, mediante test estandarizados $\mathrm{u}$ otras pruebas complementarias, tomando como referencia la sintomatología de una patología concreta que aparecerá reflejada por escrito a través de esta interacción con los chatbots.

Sin embargo, existen importantes retos a los que los chatbots deberán hacer frente en el ámbito de la entrevista tradicional clínica (Chevalier, 1997; Miller y Rollnick, 1999; Rodríguez Morejón, 1997; Rodríguez Vega, 2008): a) mantener el control sobre la entrevista (decidir quién habla y de qué), b) retomar la conducción de la entrevista, c) realizar cuestiones cerradas, abiertas y preguntas indirectas, d) adelantarse a lo que van a decir, e) avisar sobre los cambios de tema, f) pedir ejemplos, e) mantener la neutralidad, f) evitar confrontaciones innecesarias, g) mimetizar el lenguaje de las personas, h) devolverles alguna parte de lo que vamos entendiendo o comunicar nuestra impresión sobre el tema, i) evitar hacer un número excesivo de preguntas, j) controlar la impaciencia que quizás presente el interlocutor porque se encuentra bajo una fuerte tensión emocional y que cederá a medida 
que hable, k) fomentar una relación de colaboración al encontrarse el interlocutor en una zona confortable para él, 1) establecer una comunicación clara y eficiente previamente diseñada al tratar a los pacientes o sus familiares con un lenguaje cercano para que estos se muestren más comprensivos, empáticos y cooperadores y, por último $\mathrm{m}$ ) concluir la entrevista con una sintesis positiva.

Muchos de los aspectos señalados anteriormente superan en mucho las posibilidades comunicativas que proporcionan los actuales chatbots. Como se puede apreciar, la entrevista clínica necesita de una continua evaluación de la interacción por parte del terapeuta. Sin embargo, como se ha podido apreciar los bots conversacionales sociales pueden usarse para beneficiar a cualquier tipo de institución, reducir el tiempo de respuesta, proporcionar un mejor servicio al cliente, aumentar la satisfacción y aumentar la participación (Radziwill y Benton, 2017). La tecnología del chatbot supone un nuevo enfoque a la idea tradicional de entrevista clinica que puede ayudar a mejorar algunos de los procesos de intercambio de información entre logopeda y paciente, así como al establecimiento de una buena relación terapéutica basada en la motivación y en la cercanía. En los próximos años veremos cuántas de estas expectativas se van cumpliendo.

\section{REFERENCIAS}

ABASHEV, A.; GRIGORYEV, R.; GRIGORIAN, K. \& BOYKO, V. (2017): "Programming tools for messenger-based chatbot system organization: Implication for outpatient and translational medicines", BioNanoScience, 7 (2), pp. 403-407.

ABUSHAWAR, B. \& ATWELL, E. (2015): "ALICE chatbot: trials and outputs", Computación y Sistemas, 19 (4), pp. 625-632.

AUGELlO, A.; GENTILE, M. \& DIGNUM, F. (2017): "An Overview of Open-Source Chatbots Social Skills", International Conference on Internet Science. Cham: Springer, pp. 236-248.

BII, P. (2013): "Chatbot technology: A possible means of unlocking student potential to learn how to learn", Educational Research, 4 (2), pp. 218-221.

BORRAGÁN A.; DEL BORRIO J. A. y GUTIÉRREZ J. N. (1999): El juego vocal, Málaga: Aljibe.
BOSHMAF, Y.; MUSLUKHOV, I.; BEZNOSOV, K. \& RIPEANU, M. (2011): "The socialbot network: when bots socialize for fame and money", Proceedings of the 27th annual computer security applications conference, Orlando, Florida: Association for Computing Machinery, pp. 93102.

CAMERON, G.; CAMERON, D.; MEGAW, G.; BOND, R.; MULVENNA, M.; O'NEILL, S.; ARMOUR C. \& MCTEAR, M. (2017): "Towards a chatbot for digital counselling", Proceedings of the 31st British Computer Society Human Computer Interaction Conference. BCS Learning \& Development Ltd., Sunderland, UK: BCS Learning \& Development Ltd., p. 24.

CHEVALIER, A. (1997): Cómo lograr una entrevista eficaz, Barcelona: Granica.

COMENDADOR, B. E. V.; FRANCISCO, B. M. B.; MEDENILLA, 
J. S.; NACION, S. M. T. \& SERAC, T. B. E. (2016): "Pharmabot: a pediatric generic medicine consultant chatbot", Journal of Automation and Control Engineering 3 (2), pp. 137140.

D'ALFONSO, S.; SANTESTEBAN-ECHARRI, O.; RICE, S.; WADLEY, G.; LEDERMAN, R.; MILES, C. \& ALVAREZ-JIMENEZ, M. (2017): "Artificial intelligenceassisted online social therapy for youth mental health", Frontiers in psychology, 8, p. 796.

DELGADO GUERRERO, J. S.; LEÓN BAZAN, Y. Y. y SÁNCHEZ MORENO, F. J. (2017): “Desarrollo de chatbot usando bot framework de Microsoft", Espirales revista multidisciplinaria de investigación, 1 (11), pp. 52-59.

DESHPANDE, A.; SHAHANE, A.; GADRE, D.; DESHPANDE, M. \& JOSHI, P. M. A. (2017): "Survey of various chatbot implementation Techniques", International Journal of Computer Engineering and Applications, XI, online.

DEWART, H. \& SUMMERS, S. (1988): The pragmatics Profile of Early Communication Skills, Londres: NFER, Nelson.

DIVYA, S.; INDUMATHI, V.; ISHWARYA, S.; PRIYASANKARI, M. \& DEVI, K. (2018): "A Self-Diagnosis Medical Chatbot Using Artificial Intelligence", Journal of Web Development and Web Designing, 3 (1), pp. 1-7.

DOLE, A.; SANSARE, H.; HAREKAR, M. R. \& ATHALYE, M. S. (2015): "Intelligent Chat Bot for Banking System", International Journal of Emerging Trends \& Technology in Computer Science (IJETTCS), 4, 5 (2), p. 2.

EHLERS, S.; GILLBERG, C. \& WING, L. (1999): "A Screening Questionnaire for Asperger Syn- drome and other High Functioning Autism Spectrum Disorders in School Age Children", Journal of Autism and Developmental Disorders, 29, pp. 129141.

FERNÁNDEZ-ZUÑIGA, A. (2005): Guía de intervención logopédica en la tartamudez infantil, Madrid: Sintesis.

GARAYZÁBAL-HEINZE, E. (2009): "La lingüística clínica: teoría $\mathrm{y}$ práctica", Investigaciones lingüisticas en el siglo XXI, Alicante: Universidad de Alicante, pp. 131-168.

GONZÁLEZ MORENO, J. C.; RAMOS VALCÁRCEL, D. y HERNÁN FAJARDO TOR, C. (2010): "BotBlog: una propuesta de integración de bitácoras y agentes", Sistemas \& Telemática, 8 (15), pp. 51-61.

GUINOVART, J. G. (1998): "Fundamentos de Lingüística Computacional: bases teóricas, líneas de investigación y aplicaciones", Bibliodoc: anuari de biblioteconomia, documentació $i$ informació, 2, pp. 135-146.

HARVEY, P. H.; CURRIE, E.; DARYANANI, P. \& AUGUSTO, J. C. (2016): "Enhancing student support with a virtual assistant", International Conference on E-Learning, E-Education, and Online Training. Cham: Springer, pp. 101-109.

KAZI, H.; CHOWDHRY, B. S. \& MEMON, Z. (2012): "MedChatBot: An UMLS based Chatbot for Medical Students", International Journal of Computer Applications, 55 (17), pp. 1-5.

KUMAR, V. M., KEERTHANA, A.; MADHUMITHA, M.; VALLIAMMAI, S. \& VINITHASRI, V. (2016): "Snative Chatbot For Health Seekers", International Journal of Engineering And 
Computer Science, 5 (3), pp. 16022-16025.

LEÓN PÉREZ, M. A.; TAMAYO VALDÉS, M. C. y PÉREZ DUEÑAS D. (2017): "La ciencia lingüística: sugerencias epistemológicas sobre su tratamiento actual en la educación superior", Revista Conrado, 13 (59), pp. 7886.

MADHU, D.; JAIN, C. N.; SEBASTAIN, E.; SHAJI, S. \& AJAYAKUMAR, A. (2017): "A novel approach for medical assistance using trained chatbot", Inventive Communication and Computational Technologies (ICICCT), 2017 International Conference on, Coimbatore, India: IEEE, pp. 243-246.

MILLER, W. R. y ROLLNICK, S. (1999): La entrevista motivacional, Barcelona: Paidós.

MISHRA, S. K.; BHARTI, D. \& MISHRA, N. (2018): "Dr. Vdoc: A Medical Chatbot that Acts as a Virtual Doctor", Research \& Reviews: Journal of Medical Science and Technology, 6 (3), pp. 16-20.

ORACLE (2017): Chatbots 101. Oracle Corporation. Disponible en: http://www.oracle.com/us/technologies/mobile/chatbot-infographic-3672253.pdf (Fecha de consulta: 05/05/2018).

PEÑA-CASANOVA, J. (1996): Manual de Logopedia, Barcelona: Masson.

PÉREZ MONTERO, C. (1995): Evaluación del lenguaje oral en la etapa 0-6 años, Madrid: Siglo XXI Editores.

PLAZA NAVAS, P. A. (2017): Desarrollo de un sistema web para el soporte técnico remoto de primer nivel, orientado a la gestión de incidentes informáticos, basado en inteligencia artificial. Doctoral dissertation, Universidad De Guayaquil. Facultad de Cien- cias Matemáticas y Físicas. Ingenieria en Sistemas Computacionales.

POLLAK, M. \& ANDERST-KOTSIS, G. (2017): "E-mail monitoring and management with MS social bots", Proceedings of the 19th International Conference on Information Integration and Webbased Applications \& Services, Salzburg, Austria: Association for Computing Machinery, pp. 234-240.

PUYUELO M. (2000): Evaluación del lenguaje, Barcelona: Masson.

RADZIWILL, N. M. \& BENTON, M. C. (2017): "Evaluating Quality of Chatbots and Intelligent", Conversational Agents. arXiv, 1704.04579.

RANOLIYA, B. R.; RAGHUWANSHI, N. \& SINGH, S. (2017): "Chatbot for University Related FAQs", International Conference on Advances in Computing, Communications and Informatics (ICACCI), Udupi, India: IEEE, pp. 1525-1530.

RIVIERE (2004): IDEA. Inventario del espectro autista, Buenos Aires: FUNDEC.

ROBINS, D.; FEIN, D. \& BARTON, M. (2009): M-CHAT. Traducción y adaptación en España. Grupo Estudio MCHAT España. Disponible en: http://www.autismonavarra.com/wp-content/uploads/2014/10/MCHAT-R_F_Spanish_Spain.pdf (Fecha de consulta: 04/05/2018).

RODRIGUEZ BOGGIA, D. O. (2006): “¿Cómo es la entrevista con el logopeda?". Disponible en: https://www.espaciologopedico.com/revista/articulo/221/como-es-la-entrevista-con-el-logopeda.html. (Fecha de consulta: 02/11/2018).

RODRÍGUEZ MOREJÓN, A. (1997): “¿Familias poco colaboradoras 
o terapeutas poco motivadores? Técnicas de entrevista familiar en patología del lenguaje", $R e$ vista de Logopedia, Foniatría y Audiología, 17, pp. 21-37.

RODRÍGUEZ VEGA, B. (2008): Habilidades de entrevista para psicoterapeutas, Bilbao: DDB.

ROJÍ MENCHACA, M. (1999): La entrevista terapéutica, Madrid: UNED.

ROSSETI, L. (2006): The Rossetti Infant-Toddler language Scale, Linguisystems: USA.

SANDOVAL, A. M. (1998): Lingüistica computacional, Madrid: Sintesis.

SATU, M. S. \& PARVEZ, M. H. (2015): "Review of integrated applications with aiml based chatbot", Computer and Information Engineering (ICCIE), 2015 1st International Conference on, Rajshahi, Bangladesh: IEEE, pp. 87-90.

THOMAS, N. T. (2016): "An e-business chatbot using AIML and LSA",
Advances in Computing, Communications and Informatics (ICACCI), 2016 International Conference on, Jaipur, India: IEEE, pp. 2740-2742.

THORNE, C. (2017): "Chatbots for troubleshooting: A survey", Language and Linguistics Compass, 11 (10), pp. 1-8.

TRIADO, C. y FORNS, M. (1989): La evaluación del lenguaje. Una aproximación evolutiva, Barcelona: Anthropos.

WALLACE, R. S. (2009): "The anatomy of ALICE", Parsing the Turing Test, Dordrecht: Springer, pp. 181-210.

WEI, Y.; ZHU, X.; SUN, B. \& SUN, B. (2016): "Comparative studies of AIML", Systems and Informatics (ICSAI), 3rd International Conference on, Shanghai, China: IEEE, pp. 344-349.

WETHERBY A. M. \& BARRY M. P. (2002): CSBS DP, Baltimore: Paul H. Brookes Publishing Co. 